\title{
Application of the ADO method on disadvantage-factor calculation for heterogeneous slab cells considering linear anisotropic scattering effects
}

\author{
Aplicação do método ADO no cálculo do fator de desvantagem para células heterogêneas \\ planas considerando efeitos de espalhamento anisotrópico linear \\ J.F. Prolo Filho ${ }^{1 *}$; M. P. Rodrigues ${ }^{1}$ \\ ${ }^{1}$ Programa de Pós-Graduação em Engenharia Oceânica,Universidade Federal do Rio Grande,96.203-900,Rio \\ Grande-RS, Brasil \\ *joaoprolo@furg.br
}

(Recebido em 14 de abril de 2017; aceito em 22 de maio de 2017)

\begin{abstract}
Neste trabalho, o cálculo do fator de desvantagem é feito por uma versão do método de Ordenadas do Discretas Analítico (método ADO). Para isso, será considerada uma classe de problemas de transporte de nêutrons unidimensionais em geometria Cartesiana, com efeitos de espalhamento linearmente anisotrópicos em meios heterogêneos em camadas. O processo consiste em aplicar a discretização angular $\mathrm{S}_{\mathrm{N}}$, convertendo a equação de transporte integro-diferencial em um sistema de equações diferenciais ordinárias, cuja solução homogênea é obtida por um problema de autovalores quadráticos com ordem reduzida. Para estabelecer uma solução de forma fechada para esta classe de problemas, soluções particulares em termos de constantes são usadas, bem como condições de fronteira reflexivas e condições de interface. Afim de validar o código, o método e para fornecer resultados benchmark, uma classe problemas em duas camadas é tratado. Uma breve discussão sobre a influência da anisotropia é feita e aspectos numéricos são discutidos.

Palavras-chave: meio heterogêneo, espalhamento anisotropico, equação de transporte unidimensional
\end{abstract}

In this work, the disadvantage-factor calculation is performed by a version of the Analytical Discrete Ordinates method (ADO method). For this, a class of one-dimenisional neutron transport problems in Cartesian geometry, with linearly anisotropic scattering effects in layered heterogeneous media, will be considered. The process consists in applying the angular discretization $\mathrm{S}_{\mathrm{N}}$, converting the integrodifferential transport equation into an ordinary differential equations system, which homogeneous solution is obtained by a quadratic eigenvalues problem with reduced order. To establish a closed form solution for this class of problems, particular solutions in terms of constants are used, as well as reflective boundary conditions and interface conditions. In order to validate the code, the method and to provide benchmark results, a class of two-layered problems is treated. A brief discussion on the influence of anisotropy is made and numerical aspects are discussed.

Keywords: heterogeneous medium, anisotropic scattering, one-dimensional transport equation

\section{INTRODUCTION}

The disadvantage factor $(\xi)$, defined as the ratio between the average fluxes in the moderator and the fuel, is a recurring research topic in the design of heterogeneous reactors [1]. Also called shielding factor, this quantity is important for thermal utilization estimatives, mainly for criticality calculations and flux peaking evaluations.

Along the years, many authors have contributed in calculating this quantity with different approaches. Ferziger and Robinson (1965) [2], for example, used singular eigenfunctions expansions to perform disadvantage factor calculations in a slab geometry with isotropic scattering. Later, Bond and Siewert (1969) [3] extended their approach considering a second anisotropic scattering term on the moderator. More recently, Abdallah, El-Sherbiny and Sobhy (1994) [4] included the anisotropic effects also on the fuel region and applied the $\mathrm{P}_{\mathrm{N}}$ method with spatial expansion.

However, studies about the real influence of some physical parameters, such as core dimension (fuel/moderator) and anisotropy degree on the moderator region are still 
controversial issue among researchers [5,6]. Eccleston and McCormick (1970) [7], applying the Case method for an ideal problem, concluded that a second angular momentum of the scattering kernel significantly influences the disadvantage factor. On the other hand, Laletin et al. (1974) [8] obtained different results using Galerkin method, concluding that second or higher anisotropic scattering momenta were not necessary. Maiorino and Siewert (1980) [9], using $\mathrm{F}_{\mathrm{N}}$ method, also disagreed with Eccleston and McCormick in some test-cases.

Therefore, the contribution of this work will be to the study of the anisotropy influences on the disadvantage factor and on the scalar flux behavior, and to provide some benchmark results by applying of the ADO method for treating a class of one-dimensional neutron transport problems, in Cartesian geometry, considering non-multiplicative heterogeneous media with linearly anisotropic scattering. The ADO method [10] has been intensively and successfully used to find closed form solutions, in a concise and accurate way, for a wide range of onedimensional Rarefied Gas Dynamics (RGD) problems [11,12] and neutron transport problems in different geometries $[13,14,15]$. Due to the good features of this approach, including the independence from iterative schemes and the explicit solution (analytical in terms of the spatial variable), it has contributed to, in the computational point of view, a more efficient formulation. Along with these aspects, the reduced order of the derivation-associated eigenvalue problems also contribute to the low computational cost.

Thus, in the next sections, a heterogeneous version of the one-dimensional discrete ordinates neutron transport equation is introduced. Next, following the ADO formulation, a reduced order eigenvalue problem is obtained for each layer. Then, homogeneous and particular solutions are explicitly defined, completing the general solution for the proposed problems. Finally, numerical results and computational aspects are discussed.

\section{MATHEMATICAL MODEL}

In order to calculate the disadvantage factor, Barros et al. (2010) [16] and Maiorino and Siewert (1980) [9] works were used as a basis, and the discrete ordinates version for a neutron transport equation in one-dimensional Cartesian geometry, applied to a layered heterogeneous medium with linearly anisotropic scattering, in steady-state regime, is written as

$$
\begin{gathered}
\mu_{\mathrm{i}} \frac{\mathrm{d}}{\mathrm{dx}} \Psi_{\alpha}\left(\mathrm{x}, \mu_{\mathrm{i}}\right)+\sigma_{\mathrm{t}, \alpha} \Psi_{\alpha}\left(\mathrm{x}, \mu_{\mathrm{i}}\right)= \\
\frac{\sigma_{\mathrm{s} 0, \alpha}}{2} \sum_{\mathrm{k}=1}^{\mathrm{N}} w_{\mathrm{k}} \Psi_{\alpha}\left(\mathrm{x}, \mu_{\mathrm{k}}\right)+\frac{3}{2} \sigma_{\mathrm{s} 1, \alpha} \mu_{\mathrm{i}} \sum_{\mathrm{k}=1}^{\mathrm{N}} \mathrm{w}_{\mathrm{k}} \mu_{\mathrm{k}} \Psi_{\alpha}\left(\mathrm{x}, \mu_{\mathrm{k}}\right)+\mathrm{Q}_{\alpha}\left(\mathrm{x}, \mu_{\mathrm{i}}\right)
\end{gathered}
$$

with $\mathbf{i}=\mathbf{1}, \ldots, \mathbf{N}$, being $\mathbf{N}$ associated to the number of discrete directions of the Gauss-Legendre quadrature set [17], $\boldsymbol{\alpha}=\mathbf{1}, \ldots, \mathbf{M}$, where $\mathbf{M}$ corresponds to the number of layers in which the domain is subdivided, $\mathbf{x}[\mathrm{cm}]$ and $\boldsymbol{\mu}_{\mathbf{i}}$ are, respectively, the spatial and directional variables wherein the angular fluxes $\boldsymbol{\Psi}_{\boldsymbol{\alpha}}\left[\mathrm{n} / \mathrm{cm}^{2}\right.$.s $]$ are evaluated. The weights $\mathbf{w}_{\mathbf{k}}$ are associated to the Gauss-Legendre points $\boldsymbol{\mu}_{\mathrm{k}}, \mathbf{Q}_{\boldsymbol{\alpha}}\left[\mathrm{n} / \mathrm{cm}^{3}\right.$.s $]$ represents a neutron source inside the layer $\boldsymbol{\alpha}, \boldsymbol{\sigma}_{\mathrm{t}, \boldsymbol{\alpha}}$ $\left[\mathrm{cm}^{-1}\right], \boldsymbol{\sigma}_{\mathbf{s} \mathbf{0}, \boldsymbol{\alpha}}\left[\mathrm{cm}^{-1}\right]$ and $\boldsymbol{\sigma}_{\mathbf{s} \mathbf{1}, \boldsymbol{\alpha}}\left[\mathrm{cm}^{-1}\right]$ are the total, isotropic and linearly anisotropic macroscopic cross sections for the layer $\boldsymbol{\alpha}$. In particular, the scattering coefficients will be defined here as

$$
\sigma_{\mathrm{s} 0, \alpha}=\sigma_{\mathrm{t}, \alpha}-\sigma_{\mathrm{a}, \alpha}
$$

and

$$
\sigma_{\mathrm{s} 1, \alpha}=\frac{\sigma_{\mathrm{s} 0, \alpha} \beta}{3},
$$

for each layer $\boldsymbol{\alpha}$, with $\boldsymbol{\sigma}_{\mathrm{a}, \boldsymbol{\alpha}}\left[\mathrm{cm}^{-1}\right]$ being the absorption macroscopic cross section and $\boldsymbol{\beta}$ representing a Legendre coefficient in an expansion of the scattering kernel. 
For the ADO method application [10], the discrete directions are rearranged so that, for $\mathbf{i}=$ $\mathbf{1}, \ldots, \mathbf{N} / \mathbf{2}, \boldsymbol{\mu}_{\mathbf{i}}$ corresponds to the positive directions and $-\boldsymbol{\mu}_{\mathbf{i}}$ is relative to the negative directions. Consequently, Eq. (1) is subdivided in two, as

$$
\begin{gathered}
\mu_{\mathrm{i}} \frac{\mathrm{d}}{\mathrm{dx}} \Psi_{\alpha}\left(\mathrm{x}, \mu_{\mathrm{i}}\right)+\sigma_{\mathrm{t}, \alpha} \Psi_{\alpha}\left(\mathrm{x}, \mu_{\mathrm{i}}\right)= \\
\frac{\sigma_{\mathrm{s} 0, \alpha}}{2} \sum_{\mathrm{k}=1}^{\mathrm{N} / 2} \mathrm{w}_{\mathrm{k}}\left[\Psi_{\alpha}\left(\mathrm{x}, \mu_{\mathrm{k}}\right)+\Psi_{\alpha}\left(\mathrm{x},-\mu_{\mathrm{k}}\right)\right]+\frac{3}{2} \sigma_{\mathrm{s} 1, \alpha} \mu_{\mathrm{i}} \sum_{\mathrm{k}=1}^{\mathrm{N} / 2} \mathrm{w}_{\mathrm{k}} \mu_{\mathrm{k}}\left[\Psi_{\alpha}\left(\mathrm{x}, \mu_{\mathrm{k}}\right)-\Psi_{\alpha}\left(\mathrm{x},-\mu_{\mathrm{k}}\right)\right]+\mathrm{Q}_{\alpha}\left(\mathrm{x}, \mu_{\mathrm{i}}\right)
\end{gathered}
$$

and

$$
\begin{gathered}
-\mu_{\mathrm{i}} \frac{\mathrm{d}}{\mathrm{dx}} \Psi_{\alpha}\left(\mathrm{x},-\mu_{\mathrm{i}}\right)+\sigma_{\mathrm{t}, \alpha} \Psi_{\alpha}\left(\mathrm{x},-\mu_{\mathrm{i}}\right)= \\
\frac{\sigma_{\mathrm{s}, \alpha}}{2} \sum_{\mathrm{k}=1}^{\mathrm{N} / 2} \mathrm{~W}_{\mathrm{k}}\left[\Psi_{\alpha}\left(\mathrm{x}, \mu_{\mathrm{k}}\right)+\Psi_{\alpha}\left(\mathrm{x},-\mu_{\mathrm{k}}\right)\right]-\frac{3}{2} \sigma_{\mathrm{s} 1, \alpha} \mu_{\mathrm{i}} \sum_{\mathrm{k}=1}^{\mathrm{N} / 2} \mathrm{w}_{\mathrm{k}} \mu_{\mathrm{k}}\left[\Psi_{\alpha}\left(\mathrm{x}, \mu_{\mathrm{k}}\right)-\Psi_{\alpha}\left(\mathrm{x},-\mu_{\mathrm{k}}\right)\right]+\mathrm{Q}_{\alpha}\left(\mathrm{x},-\mu_{\mathrm{i}}\right),
\end{gathered}
$$

for $\mathbf{i}=\mathbf{1}, \ldots, \mathbf{N} / \mathbf{2}$ and $\alpha=\mathbf{1}, \ldots, \mathbf{M}$.

On the formulation presented here, there are neutron sources located inside some of the layers $\boldsymbol{\alpha}$, so particular solutions will be needed.

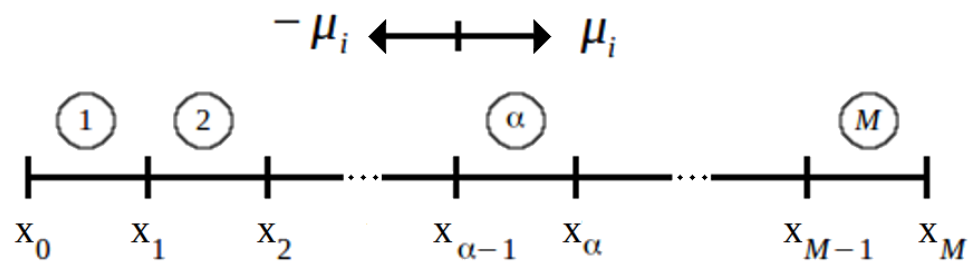

Figure 1: Description of the domain division in a heterogeneous neutron transport problem.

\section{THE ADO METHOD}

Following some basic steps of the ADO method, a homogeneous solution for the transport problem described by Eqs. (4) and (5) is proposed in the form

$$
\Psi_{\alpha}\left(\mathrm{x}, \pm \mu_{\mathrm{i}}\right)=\Phi_{\alpha}\left(v_{\alpha}, \pm \mu_{\mathrm{i}}\right) \mathrm{e}^{-\mathrm{x} / v_{\alpha}},
$$

for $\mathbf{i}=\mathbf{1}, \ldots, \mathbf{N} / \mathbf{2}, \boldsymbol{\alpha}=\mathbf{1}, \ldots, \mathbf{M}$, where the separation constant $\boldsymbol{v}_{\boldsymbol{\alpha}}$ is associated with the elementary solution $\boldsymbol{\Phi}_{\boldsymbol{\alpha}}\left(\boldsymbol{v}_{\boldsymbol{\alpha}}, \pm \boldsymbol{\mu}_{\mathrm{i}}\right)$.

This way, substituting Eq. (6) into Eqs. (4) and (5), the algebraic systems

$$
\begin{gathered}
-\frac{\mu_{\mathrm{i}}}{v_{\alpha}} \Phi_{\alpha}\left(v_{\alpha}, \mu_{\mathrm{i}}\right)+\sigma_{\mathrm{t}, \alpha} \Phi_{\alpha}\left(v_{\alpha}, \mu_{\mathrm{i}}\right)= \\
\frac{\sigma_{s 0, \alpha}}{2} \sum_{\mathrm{k}=1}^{N / 2} \mathrm{w}_{\mathrm{k}}\left[\Phi_{\alpha}\left(v_{\alpha}, \mu_{\mathrm{k}}\right)+\Phi_{\alpha}\left(v_{\alpha}-\mu_{\mathrm{k}}\right)\right]+\frac{3}{2} \sigma_{s 1, \alpha} \mu_{\mathrm{i}} \sum_{\mathrm{k}=1}^{\mathrm{N} / 2} \mathrm{w}_{\mathrm{k}} \mu_{\mathrm{k}}\left[\Phi_{\alpha}\left(v_{\alpha}, \mu_{\mathrm{k}}\right)-\Phi_{\alpha}\left(v_{\alpha},-\mu_{\mathrm{k}}\right)\right]
\end{gathered}
$$

and

$$
\begin{gathered}
\frac{\mu_{\mathrm{i}}}{v_{\alpha}} \Phi_{\alpha}\left(v_{\alpha}-\mu_{\mathrm{i}}\right)+\sigma_{\mathrm{t}, \alpha} \Phi_{\alpha}\left(v_{\alpha}-\mu_{\mathrm{i}}\right)= \\
\frac{\sigma_{\mathrm{s} 0, \alpha}}{2} \sum_{\mathrm{k}=1}^{N / 2} \mathrm{w}_{\mathrm{k}}\left[\Phi_{\alpha}\left(v_{\alpha}, \mu_{\mathrm{k}}\right)+\Phi_{\alpha}\left(v_{\alpha},-\mu_{\mathrm{k}}\right)\right]-\frac{3}{2} \sigma_{\mathrm{s} 1, \alpha} \mu_{\mathrm{i}} \sum_{\mathrm{k}=1}^{N / 2} \mathrm{w}_{\mathrm{k}} \mu_{\mathrm{k}}\left[\Phi_{\alpha}\left(v_{\alpha}, \mu_{\mathrm{k}}\right)-\Phi_{\alpha}\left(v_{\alpha},-\mu_{\mathrm{k}}\right)\right],
\end{gathered}
$$

for $\mathbf{i}=\mathbf{1}, \ldots, \mathbf{N} / \mathbf{2}$ and $\boldsymbol{\alpha}=\mathbf{1}, \ldots, M$ are obtained.

Now, two auxiliary functions are defined as

$$
\begin{aligned}
& \mathrm{U}_{\alpha}\left(v_{\alpha}, \mu_{\mathrm{i}}\right)=\Phi_{\alpha}\left(v_{\alpha}, \mu_{\mathrm{i}}\right)+\Phi_{\alpha}\left(v_{\alpha},-\mu_{\mathrm{i}}\right), \\
& \mathrm{V}_{\alpha}\left(v_{\alpha}, \mu_{\mathrm{i}}\right)=\Phi_{\alpha}\left(v_{\alpha}, \mu_{\mathrm{i}}\right)-\Phi_{\alpha}\left(v_{\alpha},-\mu_{\mathrm{i}}\right),
\end{aligned}
$$


such that, if Eqs. (7) and (8) are added, the expression

$$
\mathrm{V}_{\alpha}\left(v_{\alpha}, \mu_{\mathrm{i}}\right)=\frac{v_{\alpha}}{\mu_{\mathrm{i}}}\left[\sigma_{\mathrm{t}, \mathrm{\alpha}} \mathrm{U}_{\alpha}\left(v_{\alpha}, \mu_{\mathrm{i}}\right)-\sigma_{\mathrm{s} 0, \alpha} \sum_{\mathrm{k}=1}^{\mathrm{N} / 2} \mathrm{w}_{\mathrm{k}} \mathrm{U}_{\alpha}\left(v_{\alpha}, \mu_{\mathrm{k}}\right)\right]
$$

is obtained.

Now, subtracting Eq. (8) from Eq. (7), another relation between $\mathbf{U}_{\boldsymbol{\alpha}}\left(\boldsymbol{v}_{\boldsymbol{\alpha}}, \mu_{\mathbf{i}}\right)$ and $\mathbf{V}_{\boldsymbol{\alpha}}\left(\boldsymbol{v}_{\boldsymbol{\alpha}}, \mu_{\mathbf{i}}\right)$ is obtained, and it is given by

$$
-\frac{\mu_{\mathrm{i}}}{v_{\alpha}} \mathrm{U}_{\alpha}\left(v_{\alpha}, \mu_{\mathrm{i}}\right)+\sigma_{\mathrm{t}, \mathrm{\alpha}} \mathrm{V}_{\alpha}\left(v_{\alpha}, \mu_{\mathrm{i}}\right)=3 \sigma_{\mathrm{s} 1, \alpha} \mu_{\mathrm{i}} \sum_{\mathrm{k}=1}^{\mathrm{N} / 2} \mathrm{w}_{\mathrm{k}} \mu_{\mathrm{k}} \mathrm{V}_{\alpha}\left(v_{\alpha}, \mu_{\mathrm{k}}\right)
$$

From Eqs. (11) and (12), after some algebraic manipulations, an eigenvalue problem in terms of $\mathbf{U}_{\boldsymbol{\alpha}}\left(\boldsymbol{v}_{\boldsymbol{\alpha}}, \mu_{\mathbf{i}}\right)$ is written in the form

$$
\frac{1}{v_{\alpha}^{2}} \mathrm{U}_{\alpha}\left(v_{\alpha}, \mu_{\mathrm{i}}\right)=\frac{\sigma_{\mathrm{t}, \alpha}^{2}}{\mu_{\mathrm{i}}^{2}} \mathrm{U}_{\alpha}\left(v_{\alpha}, \mu_{\mathrm{i}}\right)-\sum_{\mathrm{k}=1}^{\mathrm{N} / 2} \mathrm{w}_{\mathrm{k}}\left[\frac{\sigma_{\mathrm{t}, \alpha} \sigma_{\mathrm{s} 0, \alpha}}{\mu_{\mathrm{i}}^{2}}+3 \sigma_{\mathrm{t}, \alpha} \sigma_{\mathrm{s} 1, \alpha}-3 \sigma_{\mathrm{s} 0, \alpha} \sigma_{\mathrm{s} 1, \alpha}\left(\sum_{\mathrm{j}=1}^{\mathrm{N} / 2} \mathrm{w}_{\mathrm{j}}\right)\right] \mathrm{U}_{\alpha}\left(v_{\alpha}, \mu_{\mathrm{k}}\right),
$$

for $\mathbf{i}=\mathbf{1}, \ldots, \mathbf{N} / \mathbf{2}$ and $\boldsymbol{\alpha}=\mathbf{1}, \ldots, \mathbf{M}$. The matrix representation of Eq. (13) is given by

$$
\left[\mathrm{D}_{\alpha}-\mathrm{A}_{\alpha}\right] \overrightarrow{\mathrm{U}_{\alpha}}=\lambda_{\alpha} \overrightarrow{\mathrm{U}_{\alpha}},
$$

where $\overrightarrow{\mathbf{U}_{\boldsymbol{\alpha}}}$ is a vector with components $\mathbf{U}_{\boldsymbol{\alpha}}\left(\boldsymbol{v}_{\boldsymbol{\alpha}}, \mu_{\mathrm{i}}\right)$, and

$$
\lambda_{\alpha}=\frac{1}{v_{\alpha}^{2}}
$$

The $\mathbf{N} / \mathbf{2} \times \mathbf{N} / \mathbf{2}$ matrices in Eq. (14) are such that

$$
\mathrm{D}_{\alpha}=\operatorname{diag}\left[\frac{\sigma_{\mathrm{t}, \alpha}^{2}}{\mu_{1}^{2}}, \frac{\sigma_{\mathrm{t}, \alpha}^{2}}{\mu_{2}^{2}}, \ldots, \frac{\sigma_{\mathrm{t}, \alpha}^{2}}{\mu_{\mathrm{N} / 2}^{2}}\right]
$$

and

$$
\mathrm{A}_{\alpha}(\mathrm{i}, \mathrm{k})=\mathrm{w}_{\mathrm{k}}\left[\frac{\sigma_{\mathrm{t}, \alpha} \sigma_{s 0, \alpha}}{\mu_{\mathrm{i}}^{2}}+3 \sigma_{\mathrm{t}, \alpha} \sigma_{\mathrm{s} 1, \alpha}-3 \sigma_{\mathrm{s} 0, \alpha} \sigma_{\mathrm{s} 1, \alpha}\left(\sum_{\mathrm{j}=1}^{\mathrm{N} / 2} \mathrm{w}_{\mathrm{j}}\right)\right],
$$

for $\mathbf{i}, \mathbf{k}=\mathbf{1}, \ldots, \mathbf{N} / \mathbf{2}$ and $\alpha=1, \ldots, M$.

With the eigenvalue problem solved, the values of $\boldsymbol{\lambda}_{\boldsymbol{\alpha}, \mathbf{j}}$ for $\mathbf{j}=\mathbf{1}, \ldots, \mathbf{N} / \mathbf{2}$ are obtained, such that the separation constants $\boldsymbol{v}_{\boldsymbol{\alpha}, \mathbf{j}}$ are found by Eq. (15) and, from Eqs. (9) and (10), the elementary solution can be written as

$$
\Phi_{\alpha}\left(v_{\alpha, j}, \mu_{\mathrm{i}}\right)=\frac{1}{2}\left[\mathrm{U}_{\alpha}\left(v_{\alpha, \mathrm{j}}, \mu_{\mathrm{i}}\right)+\mathrm{V}_{\alpha}\left(v_{\alpha, \mathrm{j}}, \mu_{\mathrm{i}}\right)\right]
$$

and

$$
\Phi_{\alpha}\left(v_{\alpha, j},-\mu_{\mathrm{i}}\right)=\frac{1}{2}\left[\mathrm{U}_{\alpha}\left(v_{\alpha, \mathrm{j}}, \mu_{\mathrm{i}}\right)-\mathrm{V}_{\alpha}\left(v_{\alpha, j}, \mu_{\mathrm{i}}\right)\right],
$$

for $\mathbf{i}, \mathbf{j}=\mathbf{1}, \ldots, \mathbf{N} / \mathbf{2}$ and $\alpha=\mathbf{1}, \ldots, \mathbf{M}$. 
Since the separation constants occur in pairs, $\left\{ \pm \boldsymbol{v}_{\alpha, j}\right\}$, with real values, and using the symmetry properties of the elementary solutions

$$
\begin{aligned}
& \Phi_{\alpha}\left(v_{\alpha, j}, \mu_{\mathrm{i}}\right)=\Phi_{\alpha}\left(-v_{\alpha, j},-\mu_{\mathrm{i}}\right), \\
& \Phi_{\alpha}\left(v_{\alpha, \mathrm{j},},-\mu_{\mathrm{i}}\right)=\Phi_{\alpha}\left(-v_{\alpha, \mathrm{j}}, \mu_{\mathrm{i}}\right),
\end{aligned}
$$

the homogeneous solutions for Eqs. (4) and (5), in an explicit form, are given by

$$
\Psi_{\alpha}^{\mathrm{h}}\left(\mathrm{x}, \mu_{\mathrm{i}}\right)=\sum_{\mathrm{j}=1}^{\mathrm{N} / 2} \mathrm{~A}_{\alpha, \mathrm{j}} \Phi_{\alpha}\left(v_{\alpha, j}, \mu_{\mathrm{i}}\right) \mathrm{e}^{-\left(\mathrm{x}-\mathrm{x}_{\alpha-1}\right) / v_{\alpha, \mathrm{j}}}+\mathrm{A}_{\alpha, \mathrm{j}+\mathrm{N} / 2} \Phi_{\alpha}\left(-v_{\alpha, j}, \mu_{\mathrm{i}}\right) \mathrm{e}^{-\left(\mathrm{x}_{\alpha}-\mathrm{x}\right) / v_{\alpha, j}}
$$

and

$$
\Psi_{\alpha}^{\mathrm{h}}\left(\mathrm{x},-\mu_{\mathrm{i}}\right)=\sum_{\mathrm{j}=1}^{\mathrm{N} / 2} \mathrm{~A}_{\alpha, \mathrm{j}} \Phi_{\alpha}\left(v_{\alpha, \mathrm{j}},-\mu_{\mathrm{i}}\right) \mathrm{e}^{-\left(\mathrm{x}-\mathrm{x}_{\alpha-1}\right) / v_{\alpha, \mathrm{j}}}+\mathrm{A}_{\alpha, \mathrm{j}+\mathrm{N} / 2} \Phi_{\alpha}\left(-v_{\alpha, \mathrm{j}},-\mu_{\mathrm{i}}\right) \mathrm{e}^{-\left(\mathrm{x}_{\alpha}-\mathrm{x}\right) / v_{\alpha, j}}
$$

for $\mathbf{i}=\mathbf{1}, \ldots, \mathbf{N} / \mathbf{2}$ and each region $\boldsymbol{\alpha}=\mathbf{1}, \ldots, \mathbf{M}$. Here, the arbitrary constants $\mathbf{A}_{\alpha, j}$ are to be determined, and they depend on the boundary conditions and particular solutions.

It is important to observe that, in this formulation, from a set of $\mathbf{N}$ discrete ordinates equations, an eigenvalue problem of order $\mathbf{N} / \mathbf{2}$ was derived, which means a relevant gain in comparison with other similar discrete ordinates approaches, where characteristic equations or eigensystems of order $\mathbf{N}$ are obtained, for the same quadrature scheme [16]. Furthermore, the expressions for the homogeneous solutions, in terms of spatial variable, are analytical, contributing to the low computational cost and high accuracy of the method.

\section{PARTICULAR SOLUTION}

Since the problem formulated by Eq. (1) has a non-homogeneous source term, particular solutions are needed. For that, if the neutron sources were constant in each layer $\alpha$ and each direction $\pm \mu_{\mathrm{i}}$, simpler particular solutions in terms of constants can be defined. So, for $\mathrm{i}=$ $1, \ldots, N / 2$,

$$
\Psi_{\alpha}^{\mathrm{p}}\left(\mathrm{x}, \mu_{\mathrm{i}}\right)=\mathrm{B}_{\alpha, \mathrm{i}}
$$

and

$$
\Psi_{\alpha}^{\mathrm{p}}\left(\mathrm{x},-\mu_{\mathrm{i}}\right)=\mathrm{C}_{\alpha, \mathrm{i}}
$$

are taken such that, substituting them into Eqs. (4) and (5), a coupled $\mathrm{N} x \mathrm{~N}$ linear system is obtained, as follows

$$
\left[\mathrm{P}_{\alpha}-\mathrm{R}_{\alpha}\right] \overrightarrow{\mathrm{O}_{\alpha}}=\overrightarrow{\mathrm{S}_{\alpha}}
$$

where

$$
\begin{aligned}
& \mathrm{P}_{\alpha}=\operatorname{diag}\left[\sigma_{\mathrm{t}, \alpha}, \ldots, \sigma_{\mathrm{t}, \alpha}\right], \\
& \mathrm{R}_{\alpha}(\mathrm{i}, \mathrm{k})=\left[\begin{array}{ll}
{\left[\frac{\sigma_{\mathrm{s} 0, \alpha}}{2} w_{\mathrm{k}}+\frac{3}{2} \sigma_{\mathrm{s} 1, \alpha} \mu_{\mathrm{i}} \mu_{\mathrm{k}} \mathrm{w}_{\mathrm{k}}\right]} & {\left[\frac{\sigma_{\mathrm{s} 0, \alpha}}{2} \mathrm{w}_{\mathrm{k}}-\frac{3}{2} \sigma_{\mathrm{s} 1, \alpha} \mu_{\mathrm{i}} \mu_{\mathrm{k}} \mathrm{w}_{\mathrm{k}}\right]} \\
{\left[\frac{\sigma_{\mathrm{so}, \alpha}}{2} w_{\mathrm{k}}-\frac{3}{2} \sigma_{\mathrm{s} 1, \alpha} \mu_{\mathrm{i}} \mu_{\mathrm{k}} \mathrm{w}_{\mathrm{k}}\right]} & {\left[\frac{\sigma_{\mathrm{so}, \alpha}}{2} \mathrm{w}_{\mathrm{k}}+\frac{3}{2} \sigma_{\mathrm{s} 1, \alpha} \mu_{\mathrm{i}} \mu_{\mathrm{k}} \mathrm{w}_{\mathrm{k}}\right]}
\end{array}\right], \\
& \overrightarrow{\mathrm{O}_{\alpha}}=\left[\begin{array}{l}
\left.\mathrm{B} \mathrm{B}_{\alpha, \mathrm{i}}\right] \\
{\left[\mathrm{C}_{\alpha, \mathrm{i}}\right]}
\end{array}\right]
\end{aligned}
$$


and

$$
\overrightarrow{S_{\alpha}}=\left[\begin{array}{c}
{\left[Q_{\alpha}\left(x, \mu_{i}\right)\right]} \\
{\left[Q_{\alpha}\left(x,-\mu_{i}\right)\right]}
\end{array}\right]
$$

for $\mathrm{i}, \mathrm{k}=1, \ldots, \mathrm{N} / 2$ and $\alpha=1, \ldots, \mathrm{M}$.

With the linear system solved, the particular solutions are obtained and the general solutions will be given by

$$
\Psi_{\alpha}\left(\mathrm{x}, \pm \mu_{\mathrm{i}}\right)=\Psi_{\alpha}^{\mathrm{h}}\left(\mathrm{x}, \pm \mu_{\mathrm{i}}\right)+\Psi_{\alpha}^{\mathrm{p}}\left(\mathrm{x}, \pm \mu_{\mathrm{i}}\right)
$$

for $\mathrm{i}=1, \ldots, \mathrm{N} / 2$ and $\alpha=1, \ldots, \mathrm{M}$.

\section{COUPLING SYSTEM}

In order to explicitly define the solutions for the problem proposed here, the reflective boundary conditions

$$
\begin{aligned}
& \Psi_{1}\left(\mathrm{x}_{0}, \mu_{\mathrm{i}}\right)=\Psi_{1}\left(\mathrm{x}_{0},-\mu_{\mathrm{i}}\right), \\
& \Psi_{\mathrm{M}}\left(\mathrm{x}_{\mathrm{M}}, \mu_{\mathrm{i}}\right)=\Psi_{\mathrm{M}}\left(\mathrm{x}_{\mathrm{M}},-\mu_{\mathrm{i}}\right),
\end{aligned}
$$

are used for $\mathbf{i}=\mathbf{1}, \ldots, \mathbf{N} / \mathbf{2}$ and, to ensure the uniformity of the fluxes between the neighboring regions, the interface condition is given by

$$
\Psi_{\alpha}\left(\mathrm{x}_{\alpha}, \pm \mu_{\mathrm{i}}\right)=\Psi_{\alpha+1}\left(\mathrm{x}_{\alpha}, \pm \mu_{\mathrm{i}}\right)
$$

for $\mathbf{i}=\mathbf{1}, \ldots, \mathbf{N} / \mathbf{2}$ and $\alpha=\mathbf{1}, \ldots, \mathbf{M}-\mathbf{1}$.

Eqs. (32)-(34) lead to a $\mathbf{M N} \mathbf{x} \mathbf{M N}$ system which solution provides the value of all $\mathbf{A}_{\boldsymbol{\alpha}, \mathbf{j}}$ coefficients and, consequently, makes Eq. (31) become completely established. After that, relevant quantities can be computed, such as the disadvantage factor and scalar flux.

\section{NUMERICAL RESULTS AND COMPUTATIONAL ASPECTS}

For the approach presented here, a two layered test case was considered (Table 1 and Figure 2 ), representing the fuel and moderator regions of a basic reactor cell, where it was possible to compare the ADO method to other analytical and numerical methods for validation and provide some benchmark profiles. In particular, the Case method's results were used here as the principal benchmark because of its analytical features. Following Maiorino and Siewert [9], the discrete ordinates version of the disadvantage factor can be given by

$$
\xi=\frac{\mathrm{a}}{\mathrm{b}-\mathrm{a}} \frac{\int_{\mathrm{a}}^{\mathrm{b}} \sum_{0}^{\mathrm{a}} \sum_{\mathrm{k}=1}^{\mathrm{N} / 2} \mathrm{w}_{\mathrm{k}}\left[\Psi_{2}\left(\mathrm{x}, \mu_{\mathrm{k}}\right)+\Psi_{2}\left(\mathrm{x},-\mu_{\mathrm{k}}\right)\right] \mathrm{dx}}{\left.\Psi_{1}\left(\mathrm{x}, \mu_{\mathrm{k}}\right)+\Psi_{1}\left(\mathrm{x},-\mu_{\mathrm{k}}\right)\right] \mathrm{dx}}
$$

where $\alpha=1,2$ represent, respectively, the fuel and the moderator regions, while the discrete ordinates version of the scalar flux is calculated by

$$
\phi_{\alpha}(\mathrm{x})=\frac{1}{2} \sum_{\mathrm{k}=1}^{\mathrm{N} / 2} \mathrm{w}_{\mathrm{k}}\left[\Psi_{\alpha}\left(\mathrm{x}, \mu_{\mathrm{k}}\right)+\Psi_{\alpha}\left(\mathrm{x},-\mu_{\mathrm{k}}\right)\right]
$$

for each layer $\alpha$. 
Table 1: Parameters used on the solution of the proposed problem.

\begin{tabular}{lccccc}
\hline & $\mathrm{x}$ & $\sigma_{\mathrm{t}, \alpha}$ & $\sigma_{\mathrm{a}, \alpha}$ & $\beta$ & $\mathrm{Q}_{\alpha}\left(\mathrm{x}, \pm \mu_{\mathrm{i}}\right)$ \\
\hline Region 1 (fuel) & $0.0 \leq \mathrm{x} \leq \mathrm{a}$ & 0.717 & 0.32 & 0.00 & 0.00 \\
\hline Region 2 (moderator) & $\mathrm{a} \leq \mathrm{x} \leq \mathrm{b}$ & 2.33 & 0.0195 & Selected cases & $\sigma_{\mathrm{t}, 2}\left(1-\sigma_{\mathrm{s} 0,2} / \sigma_{\mathrm{t}, 2}\right)$ \\
\hline
\end{tabular}

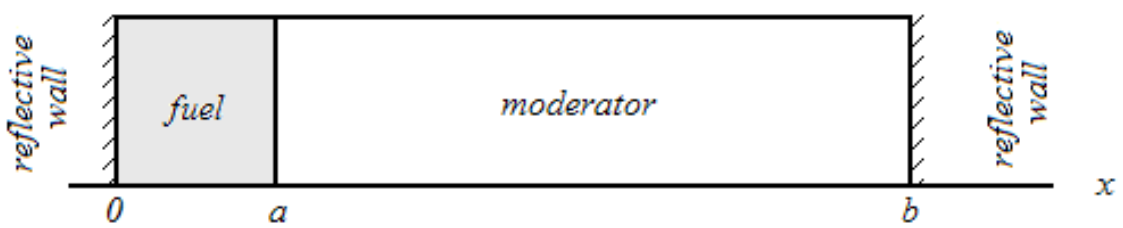

Figure 2: Description of the two-layer heterogeneous problem based on Reference [9].

Is important to observe that all the profiles were generated by a developed code in the free software Octave 4.0, using double precision computation and 80-points Gauss-Legendre quadrature set. Besides that, in terms of convergence, the four significant digits presented on Tables 2-3 for the ADO method do not change using $N>80$, ensuring the confidence of the results.

Table 2: Disadvantage factor validation to four types of cell dimensions.

\begin{tabular}{llllll}
\hline \multicolumn{1}{c}{ Computational model } & $\beta$ & $\begin{array}{c}\text { Cell 1 } \\
\mathrm{a}=0.10\end{array}$ & $\begin{array}{c}\text { Cell 2 } \\
\mathrm{a}=0.20\end{array}$ & $\begin{array}{c}\text { Cell 3 } \\
\mathrm{a}=0.30\end{array}$ & $\begin{array}{c}\text { Cell 4 } \\
\mathrm{a}=0.40 \\
\mathrm{~b}=0.90\end{array}$ \\
\hline $\mathrm{P}_{1}$ Theory [3] & 0.0 & 1.028 & 1.113 & 1.253 & 1.447 \\
Case Method [3] & 0.0 & 1.0978 & 1.2317 & 1.4077 & 1.6284 \\
$\mathrm{P}_{1}$ Spatial Approx. [4] & 0.0 & 1.1033 & 1.2311 & 1.3870 & 1.5742 \\
Asymptotic Diffusion Theory [5] & 0.0 & 1.06 & 1.18 & 1.34 & 1.56 \\
Modified ABH Method [18] & 0.0 & 1.08 & 1.20 & 1.36 & 1.58 \\
$\mathrm{~S}_{8}$ Method [18] & 0.0 & 1.09 & 1.23 & 1.43 & 1.64 \\
Integral Transport Theory [19] & 0.0 & 1.0979 & 1.2318 & 1.408 & 1.629 \\
ADO - S80 (this work) & 0.0 & 1.0976 & 1.2316 & 1.4075 & 1.6283 \\
\hline $\mathrm{P}_{1}$ Theory [3] & 0.1 & 1.027 & 1.110 & 1.245 & 1.433 \\
Case Method [3] & 0.1 & 1.0970 & 1.2283 & 1.4001 & 1.6151 \\
$\mathrm{P}_{1}$ Spatial Approx. [4] & 0.1 & 1.1026 & 1.2285 & 1.3812 & 1.5638 \\
ADO - S80 (this work) & 0.1 & 1.0967 & 1.2282 & 1.3999 & 1.6150 \\
\hline $\mathrm{P}_{1}$ Theory [3] & 0.3 & 1.026 & 1.103 & 1.230 & 1.407 \\
Case Method [3] & 0.3 & 1.0953 & 1.2215 & 1.3849 & 1.5885 \\
$\mathrm{P}_{1}$ Spatial Approx. [4] & 0.3 & 1.1013 & 1.2233 & 1.3695 & 1.5431 \\
ADO - S80 (this work) & 0.3 & 1.0950 & 1.2213 & 1.3848 & 1.5884 \\
\hline
\end{tabular}


Table 3: Disadvantage factor validation to four types of cell dimensions.

\begin{tabular}{|c|c|c|c|c|c|}
\hline Computational model & $\beta$ & $\begin{array}{c}\text { Cell 1 } \\
\mathrm{a}=0.10 \\
\mathrm{~b}=0.45\end{array}$ & $\begin{array}{c}\text { Cell 2 } \\
\mathrm{a}=0.20 \\
\mathrm{~b}=0.90\end{array}$ & $\begin{array}{c}\text { Cell 3 } \\
\mathrm{a}=0.30 \\
\mathrm{~b}=1.35\end{array}$ & $\begin{array}{c}\text { Cell 4 } \\
a=0.40 \\
b=1.80\end{array}$ \\
\hline $\mathrm{P}_{1}$ Theory [3] & 0.6 & 1.023 & 1.093 & 1.207 & 1.366 \\
\hline Case Method [3] & 0.6 & 1.0927 & 1.2113 & 1.3621 & 1.5485 \\
\hline $\mathrm{P}_{1}$ Spatial Approx. [4] & 0.6 & 1.0994 & 1.2156 & 1.3521 & 1.5121 \\
\hline $\mathrm{ADO}-\mathrm{S}_{80}$ (this work) & 0.6 & 1.0924 & 1.2111 & 1.3620 & 1.5484 \\
\hline $\mathrm{P}_{1}$ Theory $[3]$ & 0.9 & 1.021 & 1.082 & 1.184 & 1.326 \\
\hline Case Method [3] & 0.9 & 1.0901 & 1.2010 & 1.3392 & 1.5083 \\
\hline $\mathrm{P}_{1}$ Spatial Approx. [4] & 0.9 & 1.0974 & 1.2078 & 1.3346 & 1.4810 \\
\hline $\mathrm{ADO}-\mathrm{S}_{80}$ (this work) & 0.9 & 1.0898 & 1.2009 & 1.3391 & 1.5082 \\
\hline Case Method [7] & 2.0 & - & 1.1634 & - & 1.3599 \\
\hline $\mathrm{P}_{3}$ Theory $[8]$ & 2.0 & - & 1.1114 & - & 1.3114 \\
\hline $\mathrm{P}_{1}$ Spatial Approx. [4] & 2.0 & 1.0903 & 1.1793 & 1.2706 & 1.3672 \\
\hline ADO - $S_{80}$ (this work) & 2.0 & 1.0804 & 1.1632 & 1.2549 & 1.3598 \\
\hline
\end{tabular}

Based on the Tables 2-3 and Figure 3, some considerations can be made. Firstly, the moderator anisotropy causes a reduction of the disadvantage factor, independent of the cell dimension, Figure 3 (a). Besides that, this decreasing rate depends on the thickness when comparing the moderator and the fuel regions. On the other hand, for a same moderator anisotropic level, the increasing size of the cell (fuel/moderator) leads to higher values of this factor. These effects can also be noted on the scalar fluxes results, Figures 3 (b)-(e), in the heights and flattened shape of the profiles. Thus, it can be concluded that the moderator anisotropy and the cell dimension are directly associated to the thermal efficiency of a reactor.

Comparing the profiles, the Tables 2-3 makes clear that the values obtained by the ADO method are closer to the Case method, which relative error does not exceed $0.03 \%$. While in the other references the agreement among themselves is around one or two significant digits and, when compared to the Case method, they may have relative errors greater than $12.0 \%$.

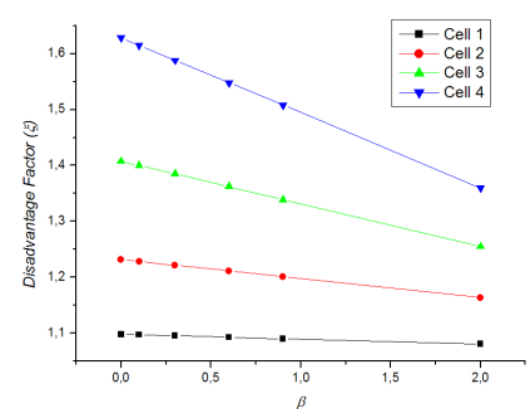

a) Disadvantage factor for all cells.

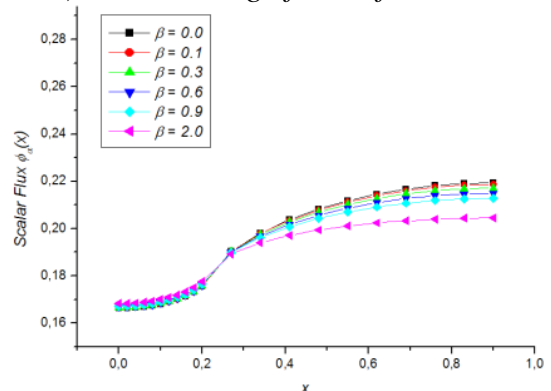

c) Scalar flux profiles for Cell 2.

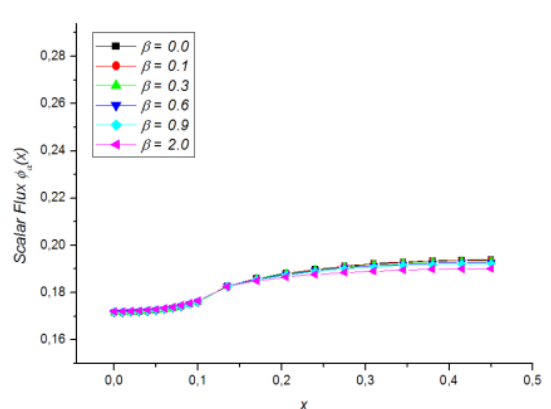

b) Scalar flux profiles for Cell 1.

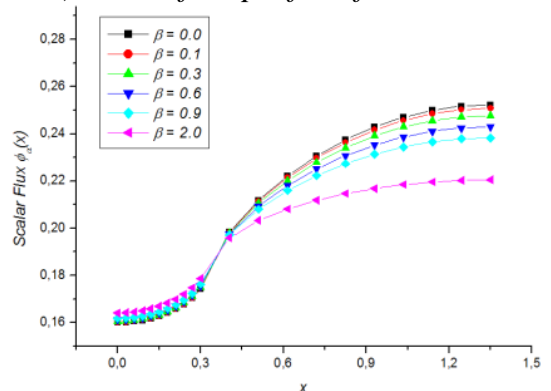

d) Scalar flux profiles for Cell 3 . 


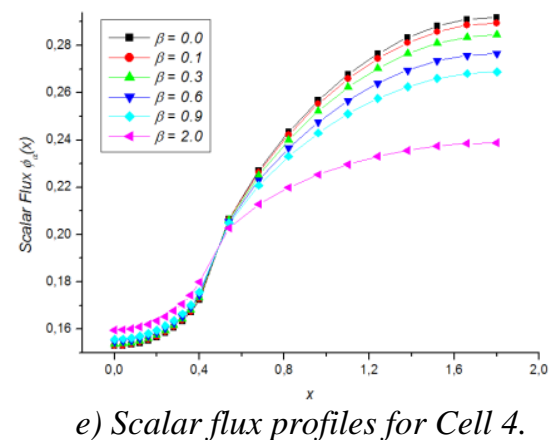

Figure 3: Profiles computed by the ADO method (this work) using $S_{80}$ quadrature scheme, considering the variation of some parameters.

\section{CONCLUSIONS}

The present work shows the ADO method as a good alternative on the solution of a class of heterogeneous neutron transport problems, in one-dimensional Cartesian geometry, where it was possible to compare some results with the available literature and provide some benchmark profiles.

On the results presented in this study, the performance of ADO method can be identified by the good agreement with the Case method (up to three significant digits and relative error lower than $0.03 \%$ ) compared to other referred formulations in the literature. Also, the influence of some parameters could be evaluated on the process, such as the effect of the anisotropy coefficient on the behavior of the disadvantage factor and scalar flux profiles.

Here, some good features of the ADO method can be highlighted: hence it doesn't use a computational spatial grid to evaluate the angular fluxes, the calculations can be made without iterative schemes, making the computational effort relatively low and spending less than $2 \mathrm{~s}$ (in a $3.10 \mathrm{GHz}$ Intel Core 15 processor with $8 \mathrm{~GB}$ of RAM) for each profile. Part of this performance was also due to the reduced order eigenvalues systems and the explicit form of the solutions (analytical in terms of the spatial variable) even in a layered domain. The code, which implementation is simple, was developed making use of the free software Octave 4.0, accepting arbitrary quadrature schemes, and working with any quadrature order. Besides that, all involved systems are well-conditioned and the parameters that arise during the process are real, making the use of complex variable techniques be unnecessary.

Thus, the objectives proposed in this work can be considered achieved, as it was possible to provide closed form solutions to the proposed problem in a concise and accurate way, showing profiles with compatible physical behavior in terms of parameters, proposal of solutions and boundary conditions used.

\section{ACKNOWLEDGEMENT}

The authors would like to thank CAPES for master's degree's scholarship and FAPERGS for partial finatial support.

\section{REFERENCES}

1. Kobayashi S. Measurement and analysis of thermal disadvantage factor for $\mathrm{UO} 2-\mathrm{H} 2 \mathrm{O}$ lattices. Journal of Nuclear Science and Technology. 1966 May;3(9):363-72.

2. Ferziger J, Robinson A. A transport theoretic calculation of the disadvantage factor. Nuclear Science and Engineering. 1965 March;21(3):382-89.

3. Bond GR, Siewert CE. The effect of linearly anisotropic neutron scattering on disadvantage factor calculations. Nuclear Science and Engineering. 1969;35(1):277-82. 
4. Abdallah AM, El-Sherbiny EM, Sobhy M. Calculation of the thermal utilization factor in a heterogeneous slab cell scattering neutrons anisotropically. Proceedings of the Second Arab Conference on the Peaceful Uses of Atomic Energy. 1994 Nov;65-75.

5. Pomraning GC, Clark M. A new asymptotic diffusion theory. Nuclear Science and Engineering. 1963 Oct;17(2):227-33.

6. Fukai Y. On the disadvantage factor in a closely packed cylindrical lattice. Journal of Nuclear Science and Technology. 1966 Apr;3(4):165-68.

7. Eccleston GW, McCormick NJ. One-speed transport disadvantage factor calculations for general anisotropic scattering. Journal of Nuclear Energy. 1970 Feb;24(1):23-34, doi:10.1016/00223107(70)90003-1.

8. Laletin NI, Sultanov NV, Vlasov YuA, Koniaev SI. The effect of the anisotropic scattering on the thermal utilization factor. Annals of Nuclear Science and Engineering. 1974 May;1(5):333-38, doi:10.1016/0302-2927(74)90014-2.

9. Maiorino JR, Siewert CE. On multi-media calculations in the theory of neutron diffusion. Annals of Nuclear Energy. 1980;7(1):535-539, doi:0.1016/0306-4549(80)90086-9.

10. Barichello LB, Siewert CE. A discrete-ordinates solution for a non-grey model with complete frequency redistribution. Journal of Quantitative Spectroscopy \& Radiative Transfer. 1999 Aug;62(6):645-675, doi:10.1016/S0022-4073(98)00096-X.

11. Scherer CS, Prolo Filho JF, Barichello LB. An analytical approach to the unified solution of kinetic equations in rarefied gas dynamics. I. Flow problems. Z Angew Math Phys. 2008 July;60(1):70-115, doi:10.1007/s00033-008-7084-4.

12. Scherer CS, Prolo Filho JF, Barichello LB. An analytical approach to the unified solution of kinetic equations in rarefied gas dynamics. II. Heat transfer problems. Z Angew Math Phys. 2008 Jul;60(4):651-87, doi:10.1007/s00033-008-7113-3.

13. Barichello LB, Rodrigues P, Siewert CE. An analytical discrete-ordinates solution for dual-mode heat transfer in a cylinder. Journal of Quantitative Spectroscopy \& Radiative Transfer. 2002 Jun;73(6):583602, doi:10.1016/S0022-4073(01)00181-9.

14. Tres A, Picoloto CB, Prolo Filho JF, Da Cunha RD, Barichello LB. Explicit formulation of a nodal transport method for discrete ordinates calculations in two-dimensional fixed-source problems. Kerntechnik. 2014 April;79(2):155-62, doi:10.3139/124.110413.

15. Ferreira CES, Emmendorfer LR, Prolo Filho JF. Formulação nodal aplicada a problemas de transporte bidimensional em geometria cartesiana. Scientia Plena. 2015;11(8):1-10, doi:10.14808/sci.plena.2015.081315.

16. Barros RC, Alves Filho H, Plat GM, Oliveira FBS, Militão DS. Analytical reconstruction scheme for the coarse-mesh solution generated by the spectral nodal method for neutral particle discrete ordinates transporet model in slab geometry. Annals of Nuclear Energy. 2010 Nov;37(11):1461-66, doi:10.1016/j.anucene.2010.06.019.

17. Stroud AH., Secrest, D. Gaussian quadrature formulas. New York: Prentice-Hall Inc; 1966. 374 p.

18. Theys MH. Integral transport theory of thermal utilization factor in infinite slab geometry. Nuclear Science and Engineering. 1960 Jan;7(1):58-63.

19. Carlvik I. Calculations of neutron flux distributions by means of integral transport methods. Sweden: Aktiebolaget Atomenergi; 1967. 100 p. 